Methods KPIs between January 2017 and January 2018 were obtained from local ERS derived audit and endoscopists with KPIs below the minimum requirement were offered individual feedback by the clinical lead. Number of procedures, Polyp Detection Rate (PDR) and Caecal Intubation rate (CIR) were compared with data from colonoscopies performed between January 2019-January 2020 with data obtained from National Endoscopy Database. Opinion on individualised KPI reporting was measured across the department using Survey Monkey.

Results Nine endoscopists (seven gastroenterologists, one surgeon, and one nurse endoscopist) were offered feedback as minimum quality standards were not met, all of whom took part in the feedback process. Six endoscopists' CIR was below the minimum requirement. Three endoscopists' CIR and PDR were below the minimum requirement. Two endoscopists performing less than 10 procedures per year, elected to cease performing colonoscopy. Four endoscopists with inadequate CIR improved following feedback. 1 endoscopist with insufficient PDR improved with feedback.

11 endoscopists responded to the survey. 82\% reported checking their KPI at least annually, with the majority (45\%) feeling that this should be reported quarterly. A formal individualised KPI report was felt to be useful by $64 \%$ of respondees.

Conclusions Providing individualised feedback did help individuals' KPIs in this cohort. We have demonstrated that using the NED data KPIs can be monitored with ease. A larger study involving multiple sites would give greater power to whether this could lead to a significant improvement in outcomes. Majority of endoscopists feel that an individualised KPI report will be helpful.

\section{P8 DOES AN EDUCATIONAL VIDEO IMPROVE BOWEL PREPARATION IN PATIENTS FIRST COLONOSCOPY? A UK MULTI-CENTRE RCT}

${ }^{1}$ Thomas Archer*, ${ }^{2}$ Keith Dear, ${ }^{3}$ Stephen Foley, ${ }^{4}$ Andy Cole, ${ }^{5}$ Jervoise Andreyev, ${ }^{6}$ Waleed Fateen, ${ }^{1}$ Mo Thoufeeq, ${ }^{6}$ Adolfo Parra-Blanco. 'Sheffield Teaching Hospitals, Sheffield, UK; ${ }^{2}$ Chesterfield Royal Hospital, Chesterfield, UK; ${ }^{3}$ Sherwood Forest Hospital, Mansfield, UK; ${ }^{4}$ Derby Royal Hospital, Derby, UK; ${ }^{5}$ Lincoln County Hospital, Lincoln, UK; ${ }^{6}$ Nottingham University Hospital, Nottingham, UK

\subsection{6/gutjnl-2020-bsgcampus.83}

Introduction Colonoscopy is the gold standard for investigation of the large bowel. Adequate bowel preparation is vital to an effective procedure. A well-informed, motivated patient, who understands the process to prepare the bowel and will adhere to it, is more likely to have adequate bowel preparation. The aim of this study is to assess whether an educational video for patients undergoing colonoscopy can lead to an improvement in bowel preparation.

Methods Participants referred for their first colonoscopy and receiving Moviprep were eligible for recruitment. Those recruited, were randomised $1: 1$ to access to the educational video or the control group. All participants were also provided with standard written instructions. The educational video was developed in collaboration with Nottingham Trent University graphics department. Primary end point was adequacy of bowel preparation, defined as a Boston Bowel Preparation Scale (BBPS) of 2 or greater in each segment. BBPS was scored at the time of the examination by the

\begin{tabular}{llll} 
Abstract P8 Table 1 & \\
\hline Risk Factor & Adequate preparation & Inadequate preparation & P value \\
\hline$<3$ motions/week & $37 / 424(9 \%)$ & $13 / 89(15 \%)$ & NS \\
Diabetes Mellitus & $47 / 424(11 \%)$ & $20 / 89(22 \%)$ & $<0.05$ \\
Parkinson's disease & $8 / 424(2 \%)$ & $5 / 89(6 \%)$ & $<0.05$ \\
Cirrhosis & $7 / 424(2 \%)$ & $4 / 89(4 \%)$ & NS \\
\hline
\end{tabular}

endoscopist performing the examination. Endoscopists received training on BBPS via an online video.

Results 513 participants were recruited, from 6 centres, with 254 participants randomised to access to the education video. The mean age was 58 (range 18-88). 265 (52\%) of whom were female. 54 patients in the control group had inadequate prep, compared with 35 participants in the intervention group ( $\mathrm{p}$ value $<0.05$, CI 0.381 to 0.967 ). The rate of adequate bowel preparation was not significantly different between centres. There was no significant difference between recognised risk factors for poor bowel preparation between the two groups. The association of adequacy of bowel preparation and risk factors for poor bowel preparation is shown below.

Conclusions Many factors affect the quality of bowel preparation. This study demonstrates that an educational video leads to a greater proportion of adequate bowel preparation compared with standard instructions alone. The number needed to treat to prevent one excess inadequate bowel preparation in this study is 14 . Widespread adoption of enhanced patient education, such as this educational video, could lead to improved adequacy of bowel preparation.

\section{P9 OUTCOMES FROM THE UK PURASTAT ${ }^{\circledR}$ REGISTRY: MULTICENTRE OBSERVATIONAL STUDY OF PURASTAT ${ }^{\circledR}$ USE IN GASTROINTESTINAL BLEEDING}

${ }^{1}$ Sophie Arndtz*, ${ }^{1}$ Sharmila Subramaniam, ${ }^{1}$ Ejaz Hossain, ${ }^{1}$ Mohamed Abdelrahim ${ }^{2}$ Yeng Ang, ${ }^{3}$ losif Beintaris, ${ }^{4}$ Massimiliano di Pietro, ${ }^{5}$ Marietta lacucci, ${ }^{6}$ Brian Saunders, ${ }^{6}$ Noriko Suzuki, ${ }^{1}$ Pradeep Bhandari. 'Queen Alexandra Hospital, Portsmouth, UK; ${ }^{2}$ Salford Hospital, Salford Royal NHS Foundation Trust, UK; ${ }^{3}$ North Tees and Hartlepool NHS Foundation Trust, North Tees, UK; ${ }^{4}$ Addenbrooke's Hospital, Cambridge, UK; ${ }^{5}$ University Hospitals Birmingham NHS Foundation Trust, Birmingham, UK; ${ }^{6}$ St Mark's Hospital, Harrow, UK

\subsection{6/gutjnl-2020-bsgcampus.84}

Introduction PuraStat ${ }^{\circledR}$ is a novel haemostatic agent without the risk of thermal injury, perforation or loss of mucosal views associated with other treatments such as heat therapy, clips or haemostatic powders. Our aim was to evaluate the efficacy of PuraStat ${ }^{\circledR}$ in the prevention and treatment of gastro-intestinal bleeding.

Methods This is a prospective analysis of PuraStat ${ }^{\circledR}$ use in the UK, with 6 tertiary referral centres open to recruitment. Data was collected on procedure \& lesion details, haemostasis management and complications for endoscopies where PuraStat ${ }^{\circledR}$ was used.

Results 226 procedures were included across 3 indications: 198 high risk resection, 6 upper gastro-intestinal bleeding (UGIB) and 22 radiation proctopathy. PuraStat ${ }^{\circledR}$ was used for immediate haemostasis in 100 bleeding episodes, of which 92 were as primary agent and 8 as secondary agent (after failure of alternative initial therapy) and for prevention of delayed 